\title{
Determinants of Patient Delay in Transient Ischemic Attack
}

\author{
L. Servaas Dolmans ${ }^{\mathrm{a}}$ Arno W. Hoes ${ }^{\mathrm{a}}$ Marie-Louise E.L. Bartelink ${ }^{\mathrm{a}}$ \\ L. Jaap Kappelle ${ }^{b}$ Frans H. Rutten ${ }^{a}$ for the MIND-TIA Study Group \\ a Julius Center for Health Sciences and Primary Care, University Medical Center Utrecht, Utrecht University, \\ Utrecht, The Netherlands; ${ }^{b}$ Department of Neurology, University Medical Center Utrecht, Utrecht University, \\ Utrecht, The Netherlands
}

\section{Keywords}

Transient ischemic attack · Minor stroke · Patient delay · Determinants

\begin{abstract}
Introduction: Early diagnosis and stroke preventive treatment in patients with transient ischemic attack (TIA) are crucial, but hampered by delayed reporting of symptoms. Previous studies on causes of patient delay provided inconsistent results. We aimed to assess determinants of patient delay among patients with symptoms suggestive of TIA. Methods: We interviewed participants referred by their general practitioner to an outpatient TIA clinic within $72 \mathrm{~h}$ from symptom onset. We determined (i) the exact time from symptom onset to the first contact with a medical service (patient delay); (ii) demographic and clinical characteristics; (iii) patient's initial perception, and reaction to symptoms; and (iv) patient's knowledge about TIA. We used multivariable linear regression to identify determinants of patient delay. Results: We interviewed 202 suspected TIA patients (mean age 67.7 (SD 13.7) years, 111 (55.0\%) male), of whom 123 (60.9\%) received a definite diagnosis of TIA or minor stroke. Median patient delay was 1.5 (interquartile range $0.4-14.6$ ) hours. Of all patients, 119 (58.9\%) considered a TIA (or stroke) as the cause of their symptoms. Among them, 30 (25.2\%) thought it was
\end{abstract}

a medical emergency, while of the 83 not considering TIA as the cause of symptoms $38(45.8 \%)$ thought of a medical emergency. Independently related to increased delay were (i) symptom onset out of hours, (ii) absence of dysarthria, (iii) being unaware that TIA requires urgent treatment, (iv) not considering the event an emergency, and ( $\mathrm{v}$ ) knowledge of TIA symptoms. Results for patients with a definite diagnosis of TIA/minor stroke were similar to those with alternative diagnoses. Conclusion: Patients still tend to wait till office hours to report TIA symptoms. Speech difficulties, and specifically dysarthria, are related to shorter delay. To reduce patient delay, awareness of TIA symptoms should increase and more importantly lay people should be educated to consider a TIA an emergency.

C 2019 The Author(s)
Published by S. Karger AG, Basel

\section{Introduction}

A transient ischemic attack (TIA) is characterized by short-lasting and often mild signs and symptoms, which easily results in trivialization or misinterpretation. Moreover, the clinical manifestations of TIA vary strongly and can resemble many other conditions. Patients should, however, report symptoms suggestive of a TIA as soon as

\begin{tabular}{|c|c|}
\hline KARGER & $\begin{array}{l}\text { (c) } 2019 \text { The Author(s) } \\
\text { Published by S. Karger AG, Basel }\end{array}$ \\
\hline $\begin{array}{l}\text { E-Mail karger@karger.com } \\
\text { www.karger.com/ene }\end{array}$ & $\begin{array}{l}\text { This article is licensed under the Creative Commons Attribution- } \\
\text { NonCommercial-NoDerivatives } 4.0 \text { International License (CC BY- } \\
\text { NC-ND) (http://www.karger.com/Services/OpenAccessLicense). } \\
\text { Usage and distribution for commercial purposes as well as any dis- } \\
\text { tribution of modified material requires written permission. }\end{array}$ \\
\hline
\end{tabular}

L. Servaas Dolmans, MD

Julius Center for Health Sciences and Primary Care

University Medical Center Utrecht, Utrecht University

PO Box 85500, Stratenum 6, NL-131 3508 GA Utrecht (The Netherlands)

E-Mail L.S.Dolmans@ umcutrecht.nl 
possible. A rapid diagnostic assessment followed by an early start of stroke preventive treatment in those with a confirmed diagnosis is crucial to keep the risk of a subsequent ischemic stroke as low as possible [1-3]. However, previous studies showed that there is substantial patient delay; $30-40 \%$ of patients delay contacting a medical service for $>24 \mathrm{~h}$ [4].

Little is known about the determinants of this patient delay. In a study from the United Kingdom, delay among TIA or minor stroke patients before contacting a general practitioner (GP) was much longer during out of office hours than during office hours ( 24.8 vs. $4.0 \mathrm{~h}$ ) [5]. Three quantitative studies that aimed to assess potential determinants of patient delay among patients with a neurologist's diagnosis of TIA or minor stroke showed conflicting results; for example, recognition of symptoms was inconsistently associated with delay [6-8]. These studies had 2 important limitations. First, these studies only applied univariable analyses and the independent contribution of individual determinants to delay was not assessed. Second, the studies only included those with established TIA or minor stroke. Multivariable analyses are needed to better quantify different determinants of patient delay, preferably in the domain of diagnostic interest and from the perspective of the patient, that is, patients suspected of TIA.

A qualitative interview study among 20 TIA patients from the UK reported that patients' recognition of typical stroke symptoms could result in urgent action by patients if symptoms are more severe, but on the other hand could result in delay if symptoms are non-severe or vague [9].

We aimed to assess determinants of patient delay with a multivariable quantitative approach among patients who were referred to an outpatient TIA clinic with symptoms suggestive of TIA.

\section{Materials and Methods}

This study was part of the MIND-TIA (Markers in the Diagnosis of transient ischemic attack) study, designed to determine the (added) value of serum biomarkers in the diagnosis of TIA [10]. In total, 206 patients suspected of a TIA by their GP were recruited from October 2013 to October 2016. A research nurse visited participants within $72 \mathrm{~h}$ from the onset of symptoms and standardized history was taken using a prespecified questionnaire that also included questions on patient delay.

Exclusion criteria were (i) the presence of ongoing symptoms during GP consultation, that is, suspicion of an ongoing stroke, (ii) severe cognitive impairment or insufficient knowledge of the Dutch language, and (iii) a life expectancy of $<6$ months. Additionally, we excluded patients if they had already sought medical help in response to symptoms that preceded the episode that was assessed, and we were thus unable to determine patient delay.

The standardized questionnaire (added as online supplementary File (see www.karger.com/doi/10.1159/000501077) included the following items: (1) the exact time from onset of symptoms to the first contact with a medical service (patient delay); (2) demographic characteristics; (3) the onset, type and duration of signs and symptoms; (4) the initial patient's response to signs and symptoms (what did the patient do?); (5) the initial patient's perception (what did the patient think about the cause of their symptoms and its severity? Was it considered to be an emergency?); (6) the general knowledge about the disease TIA (does the patient know (i) which symptoms and signs may be provoked by a TIA, and (ii) that TIA is a precursor of stroke?).

A panel of 3 neurologists made a definite diagnosis, differentiating TIA or minor stroke from alternative diagnoses based on all available diagnostic information, including brain imaging and 6 months of follow-up. The follow-up period, providing information on possible additional cerebrovascular events or new symptoms that put the initial event in a different perspective, was used to assist the panel in deciding whether at the time of presentation a TIA was present [11]. The panel applied the time-based diagnosis of TIA to discriminate TIA from minor stroke.

Delay is presented as median with $25-75 \%$ interquartile range (IQR). We used linear regression analyses to investigate the relation between patient delay and potential determinants. Delay was logarithmically transformed because of its skewed distribution. We defined "correct knowledge of TIA" as being aware of (i) key symptoms and signs provoked by a TIA and (ii) TIA being a precursor of stroke. Multivariable analyses applying stepwise backward selection (using a cutoff of $p<0.05$ ) were performed (i) for the total study population of patients suspected of TIA and (ii) separately for those patients with a definite diagnosis of TIA or minor stroke according to the panel.

\section{Results}

We included 202 of in total 206 participants, 2 patients did not complete the survey on delay and 2 patients were excluded because the GP was consulted because of symptoms that preceded the suspected TIA that was assessed. Table 1 shows the characteristics of the 202 participants. Mean age was 67.7 (SD 13.7) years, $111(55.0 \%)$ were male, and the expert panel classified $60.9 \%$ of cases as TIA $(n=102)$ or minor stroke $(n=21)$. On average, the interview by the research nurse took place 48.0 (IQR 28.1-58.0) h after symptom onset.

The median patient delay of all 202 patients was 1.5 (IQR 0.4-14.6) hours. In the 123 patients with a definite diagnosis of TIA or minor stroke this was similar; 1.5 (IQR 0.3-14.5) hours. In 102 (50.5\%) patients, symptoms occurred during out of office hours, and the median patient delay in this subgroup was 9.0 (IQR 0.73- 
Table 1. Patient characteristics of 202 patients suspected of TIA by their GP and referred to the TIA outpatient clinic

\begin{tabular}{lc}
\hline Characteristics & Total $(n=202)$ \\
\hline Age in years, mean (SD) & $67.7(13.7)$ \\
Gender, male, $n(\%)$ & $111(55.0)$ \\
History of TIA or ischemic stroke, $n(\%)$ & $45(22.3)$ \\
Living situation, $n(\%)$ & \\
$\quad$ Alone & $52(25.7)$ \\
With a partner & $145(71.8)$ \\
In a nursing home & $5(2.5)$ \\
Onset of symptoms out of hours, $n(\%)$ & $102(50.5)$ \\
Weekend days & $47(23.3)$ \\
Weekdays out of hours & $55(27.2)$ \\
Duration of symptoms in hours, median (IQR) & $0.4(0.2-1.5)$ \\
Symptoms, $n$ (\%) & \\
Motor & $83(41.1)$ \\
Sensory & $85(42.1)$ \\
Visual & $63(31.2)$ \\
$\quad$ Blurred vision & $23(11.4)$ \\
$\quad$ Diplopia & $16(7.9)$ \\
$\quad$ Hemianopsia & $14(6.9)$ \\
$\quad$ Monocular loss of vision & $10(5.0)$ \\
Communication & $97(48.0)$ \\
Dysarthria & $38(18.8)$ \\
Dysphasia & $59(29.2)$ \\
Diagnosis according to expert panel, $n(\%)$ & $123(60.9)$ \\
TIA or minor stroke & $79(39.1)$ \\
Alternative diagnoses &
\end{tabular}

* Patients may experience multiple symptoms.

TIA, transient ischemic attack; IQR, interquartile range.

17.6) hours, compared to 0.8 (IQR 0.3-2.3) hours in the 100 patients with symptoms occurring during office hours.

In $80.7 \%$, the first contacted health-care provider was the GP during office hours, in $16.8 \%$, it was the GP outof-hours service, and $2.5 \%$ of patients directly contacted the ambulance service or directly visited the hospital emergency department.

Sixty-eight $(33.7 \%)$ patients interpreted their symptoms as a medical emergency, $58.9 \%$ considered the possibility of a TIA/stroke as the underlying cause of their symptoms, $47 \%$ had correct general knowledge about TIA, and $82.7 \%$ considered it important that a TIA is treated urgently.

Table 2 provides the results of the univariable linear regression analyses, for both the total study population and selectively for the 123 patients with a definite diagnosis of TIA/minor stroke. Age and sex were not related to delay. The results for the larger population show 8 variables with a beta coefficient with a $p$ value $<0.10$. Related to increased delay were (i) a negative family history of cardiovascular disease $<65$ years, (ii) general knowledge of TIA, (iii) not being aware that TIA requires urgent treatment, (iv) symptom onset during the weekend and (v) out of hours in general, (vi) communication problems, and specifically (vii) dysarthria, and (viii) not considering the event to be an emergency. Overall, the results for the subgroup of TIA/minor stroke patients $(n=123)$ were very similar. Particularly the aforementioned variables showed comparable beta coefficients.

The final concise multivariable model for the total study population is shown in Table 3. In the final model, 5 variables remained independently related to patient delay: (i) absence of dysarthria, (ii) onset of symptoms out of hours, (iii) the patient being unaware that a TIA requires urgent treatment, (iv) not considering the event to be an emergency, and (v) general knowledge of TIA symptoms. The final multivariable model for only TIA and minor stroke patients (also in Table 3 ) consists of 3 of these 5 variables (with consistent beta coefficients): (i) dysarthria, (ii) onset out of hours, and (iii) considering the event an emergency.

Additional analyses showed no association between considering the event a medical emergency and general knowledge about TIA. There was a relation between considering a TIA as the cause of symptoms and the sense of experiencing a medical emergency; however, this was a negative association. Among the patients who thought they could actually have had a TIA, 30 of $119(25.2 \%)$ considered it a medical emergency, versus 38 of 83 (45.8\%) patients who did not consider a TIA (RR 0.55 [0.37$0.81]$ ). Among the 123 patients who showed to have a TIA/minor stroke this was similar: 27.3 vs. $43.5 \%$, respectively (RR 0.63 [0.38-1.03]). The only other variable associated (positively) to the sense of experiencing a medical emergency was being aware that a TIA requires urgent treatment.

\section{Discussion}

Our study provides relevant new insights in the potential reasons for patient delay in suspected TIA. Of the typical TIA symptoms, speech difficulties, and more specifically dysarthria, were independently related to a shorter patient delay. In previous studies, there were conflicting results about the role of patient's recognition of symptoms. We could show that patient's recognition of symp- 
Table 2. Univariable analyses of determinants of patient delay in the total study population, and in the subgroup of 123 patients with a definite diagnosis of TIA/minor stroke

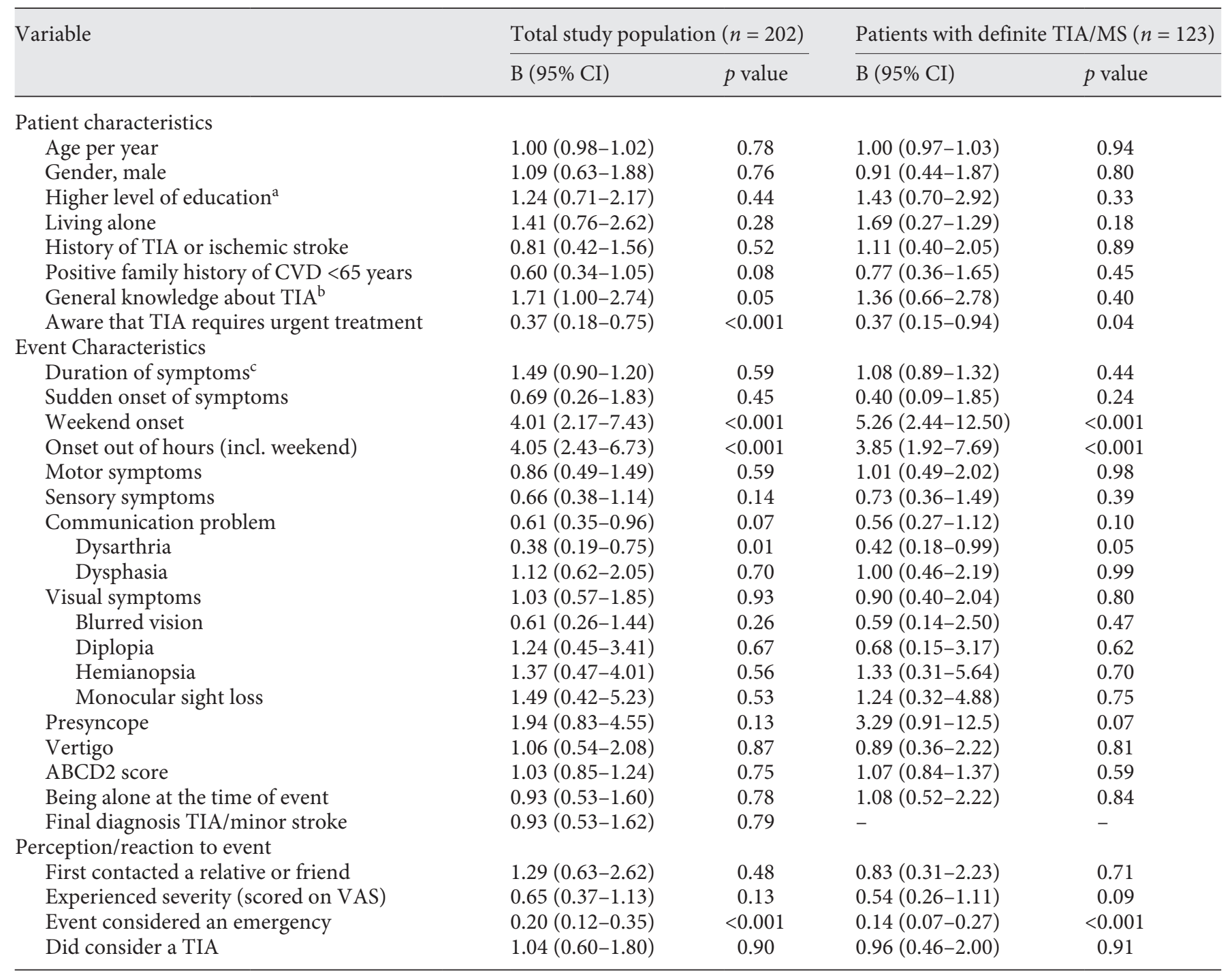

The outcome patient delay, as a continuous variable in minutes, was naturally log-transformed.

a Post-secondary education.

${ }^{\mathrm{b}}$ Knowing symptoms of TIA and aware that TIA is a precursor of stroke.

${ }^{c}$ Naturally log-transformed.

TIA, transient ischemic attack; MS, minor stroke; B, unstandardized beta coefficient; CVD, cardiovascular disease; ABCD2, prognostic score for early stroke risk prediction, including the items age, blood pressure, clinical symptoms, duration and diabetes; VAS, visual analogue scale.

toms, or general knowledge about TIA symptoms, do not necessarily lead to an urgent call for medical advice. A more important determining factor seems to be the patient's knowledge that a TIA warrants urgent treatment. Furthermore, we showed that delays are much longer during out of office hours, even in a health-care system with $24 \mathrm{~h}$ availability of GP care.
In contrast with previous studies, we analyzed a larger population of patients with symptoms suggestive of a TIA and performed a separate analysis among patients with a confirmed TIA or minor stroke. The larger population represents the clinical domain in which a quick response to symptoms is required. Moreover, as in around a quarter of referred suspected TIA cases, the consulting neu- 
Table 3. Final multivariable linear regression model of determinants of patient delay, in the 202 patients suspected of TIA, and in the subgroup of 123 patients with a definite diagnosis of TIA/minor stroke

\begin{tabular}{lcc}
\hline Variable & B (95\% CI $)$ & $p$ value \\
\hline Total study population $(n=202)$ & & 0.04 \\
Dysarthria & $0.53(0.29-0.96)$ & $<0.001$ \\
Onset of symptoms out of hours & $3.01(1.87-4.83)$ & $<0.001$ \\
Event considered an emergency & $0.25(0.15-0.42)$ & 0.04 \\
General knowledge about TIA & $1.64(1.02-2.66)$ & 0.05 \\
Aware that TIA requires urgent treatment & $0.53(0.28-0.99)$ & 0.05 \\
Patients with definite TIA/minor stroke $(n=123)$ & $0.49(0.24-1.01)$ & $<0.001$ \\
Dysarthria & $2.97(1.63-5.39)$ & $<0.001$ \\
Onset of symptoms out of hours & $0.25(0.15-0.42)$ & \\
Event considered an emergency & & \\
\hline The outcome patient delay, as a continuous variable in minutes, was naturally log-transformed because of a \\
skewed distribution.
\end{tabular}

rologist is uncertain about the final diagnosis, delay studies including only confirmed TIA patients are hampered by selection of more typical TIA cases. Interestingly, our results from both suspected TIA patients as confirmed TIA patients point out the same determinants of patient delay, with consistent beta coefficients from univariable as well as multivariable analyses.

Motor symptoms and speech difficulties have been inconsistently associated with shorter delays by TIA patients [6-8]. Unlike previous studies, we distinguished dysarthria from dysphasia, and in multivariate analyses, specifically dysarthria was an independent predictor of delay. Either this specific neurological deficit triggers patients or their relatives to seek for medical help rapidly, or dysarthria is part of a combination of symptoms that creates more urgency. We found no evidence for a relation between motor symptoms and delay. A possible explanation is that milder and short-lasting motor deficits can be easily misinterpreted or trivialized.

Earlier studies did not provide a conclusive answer regarding the role of patient's recognition of symptoms suggestive of TIA [6-9]. Our study showed that recognition of symptoms alone is not a key trigger to respond to symptoms rapidly. The participants that did consider a TIA even showed less sense of urgency than those who did not. Moreover, general knowledge of TIA (symptoms) was associated with longer instead of shorter delays. Possibly, this can be explained taking into account the comforting effect of symptoms that resolve rather quickly. In the acute stage this effect might be even stron- ger in patients that recognize a TIA, than in patients who have no clear idea what they experienced. It appears logical that knowledge about the required urgency in suspected TIA was associated with both shorter delays and the sense of urgency. However, taken all together, these data suggest a general lack of sense of urgency in lay people in case of suspected TIA.

Out of hours symptom onset shows to be a strong determinant of patient delay. Patients tend to wait until office hours to report their symptoms. This is in line with the findings by Lasserson et al. [5], who attributed the effect of time of symptom onset largely to the lack of accessible GP care out of hours. A relevant difference with this study, however, is that the Dutch health-care system nowadays includes an easily accessible round the clock GP out-of-hours service, which is commonly used by patients. Moreover, in the past decade, campaigns comparable to the UK "Act FAST" campaign have encouraged people to respond to stroke-like symptoms immediately [12]. Nevertheless, still $80 \%$ of patients reported their symptoms during routine office hours.

Compared to other studies assessing patient delay, we interviewed patients early after symptom onset, in this way limiting recall bias. Still some degree of recall bias must be considered, especially concerning our questions about knowledge of TIA. In our standardized questionnaire, we specifically asked for the knowledge prior to the suspected event; however, answers could be influenced by their search for medical advice and GP consultation. Furthermore, it is important to realize that patients with 
symptom onset $>72 \mathrm{~h}$ before contacting the GP were not included in the study.

Translating our results to clinical practice, this study highlights that beyond knowing and recognizing strokelike symptoms, lay people still need to learn to act in case of symptoms suggestive of a TIA, explicitly also if symptoms are mild and/or short lasting.

\section{Conclusion}

Patients still tend to delay till office hours to report TIA symptoms. Speech difficulties, and specifically dysarthria, are related to shorter patient delay. To reduce patient delay, awareness of TIA symptoms should increase and more importantly lay people should be educated to consider a TIA as an emergency.

\section{Statement of Ethics}

The MIND-TIA study has been approved by the Medical Research Ethics Committee of the University Medical Center of Utrecht, the Netherlands, on September 5, 2013, and was performed in accordance with the ethical standards laid down in the 1964 Declaration of Helsinki and its later amendments. Participants gave written informed consent.

\section{Disclosure Statement}

The authors have no conflicts of interest to declare.

\section{Author Contributions}

L.S.D.: is $\mathrm{PhD}$ candidate and the primary researcher. L.S.D. and F.H.: drafted the manuscript. All authors have been involved in revising it critically and approved the final manuscript.

\section{The MIND-TIA Study Group}

L.S. Dolmans, $\mathrm{MD}^{1}$; Marie-Louise E.L. Bartelink, $\mathrm{MD}, \mathrm{PhD}^{1}$; Frans H. Rutten, $\mathrm{MD}, \mathrm{PhD}^{1}$; Arno W. Hoes, $\mathrm{MD}, \mathrm{PhD}^{1}$; L. Jaap Kappelle, $\mathrm{MD}, \mathrm{PhD}^{2}$; E.J. van Dijk, $\mathrm{MD}, \mathrm{PhD}^{3}$; P.J. Nederkoorn, $\mathrm{MD}, \mathrm{PhD}^{4}$; S. van Delft, $\mathrm{PhD}^{5}$; G.J. Seppenwoolde

${ }^{1}$ Julius Center for Health Sciences and Primary Care, University Medical Center Utrecht, Utrecht University, Utrecht, The Netherlands.

${ }^{2}$ Department of Neurology, University Medical Center Utrecht, Utrecht University, Utrecht, The Netherlands.

${ }^{3}$ Department of Neurology, Radboud University Medical Center, Nijmegen, The Netherlands.

${ }^{4}$ Department of Neurology, Amsterdam UMC, University of Amsterdam, Amsterdam, The Netherlands.

${ }^{5}$ Saltro Diagnostic Center for Primary Care, Utrecht, The Netherlands.

\section{References}

1 Rothwell PM, Giles MF, Chandratheva A, Marquardt L, Geraghty O, Redgrave JN, et al.; Early use of Existing Preventive Strategies for Stroke (EXPRESS) study. Effect of urgent treatment of transient ischaemic attack and minor stroke on early recurrent stroke (EXPRESS study): a prospective populationbased sequential comparison. Lancet. 2007 Oct;370(9596):1432-42.

2 Rothwell PM, Algra A, Chen Z, Diener HC, Norrving B, Mehta Z. Effects of aspirin on risk and severity of early recurrent stroke after transient ischaemic attack and ischaemic stroke: time-course analysis of randomised trials. Lancet. 2016 Jul;388(10042): 365-75.

3 Lavallée PC, Meseguer E, Abboud H, Cabrejo L, Olivot JM, Simon O, et al. A transient ischaemic attack clinic with round-the-clock access (SOS-TIA): feasibility and effects. Lancet Neurol. 2007 Nov;6(11):953-60.

4 Dolmans LS, Hoes AW, Bartelink ME, Koenen NC, Kappelle LJ, Rutten FH. Patient delay in TIA: a systematic review. J Neurol. 2019 May;266(5):1051-8.

5 Lasserson DS, Chandratheva A, Giles MF, Mant D, Rothwell PM. Influence of general practice opening hours on delay in seeking medical attention after transient ischaemic attack (TIA) and minor stroke: prospective population based study. BMJ. 2008 Sep;337 sep18 3:a1569.

6 Giles MF, Flossman E, Rothwell PM. Patient behavior immediately after transient ischemic attack according to clinical characteristics, perception of the event, and predicted risk of stroke. Stroke. 2006 May;37(5):1254-60.

7 Chandratheva A, Lasserson DS, Geraghty OC, Rothwell PM; Oxford Vascular Study. Population-based study of behavior immediately after transient ischemic attack and minor stroke in 1000 consecutive patients: lessons for public education. Stroke. 2010 Jun;41(6):1108-14.

8 Wilson AD, Coleby D, Taub NA, Weston C, Robinson TG. Delay between symptom onset and clinic attendance following TIA and mi- nor stroke: the BEATS study. Age Ageing. 2014 Mar;43(2):253-6.

9 Mc Sharry J, Baxter A, Wallace LM, Kenton A, Turner A, French DP. Delay in seeking medical help following Transient Ischemic Attack (TIA) or "mini-stroke": a qualitative study. PLoS One. 2014 Aug;9(8):e104434.

10 Dolmans LS, Rutten FH, El Bartelink ML, Seppenwoolde G, van Delft S, Kappelle LJ, et al. Serum biomarkers for the early diagnosis of TIA: the MIND-TIA study protocol. BMC Neurol. 2015 Jul;15(1):119.

11 Bertens LC, Broekhuizen BD, Naaktgeboren CA, Rutten FH, Hoes AW, van Mourik Y, et al. Use of expert panels to define the reference standard in diagnostic research: a systematic review of published methods and reporting. PLoS Med. 2013 Oct;10(10):e1001531.

12 Flynn D, Ford GA, Rodgers H, Price C, Steen $\mathrm{N}$, Thomson RG. A time series evaluation of the FAST National Stroke Awareness Campaign in England. PLoS One. 2014 Aug;9(8): e104289. 